\title{
INTEGRAL DUBROVIN VALUATION RINGS
}

\author{
PATRICK J. MORANDI AND ADRIAN R. WADSWORTH
}

\begin{abstract}
In the preceding paper, Dubrovin valuation rings integral over their centers in central simple algebras were characterized by value functions. Here, these value functions are used to give a method for extending integral Dubrovin valuation rings in generalized crossed product algebras. Several applications of this extension theorem are given, including new and more natural proofs of some theorems on valued division algebras over Henselian fields.
\end{abstract}

\section{INTRODUCTION}

This paper is a sequel to $\left[M_{2}\right]$. In previous papers $\left[D_{1}, D_{2}, B G, W_{1}\right]$ a strong case has been made that Dubrovin valuation rings are a very good generalization to central simple algebras of the classical valuation rings on fields and division algebras. While Dubrovin valuation rings do not come equipped with a valuation, it was shown in $\left[\mathrm{M}_{2}\right]$ that there is a value function associated to every Dubrovin valuation ring integral over its center. We give here several applications of this result which use value functions in order to construct integral Dubrovin valuation rings. These lead to generalizations (with much better proofs) of some theorems from [ $\mathrm{JW}$ and $\mathrm{W}_{2}$ ].

The arguments given here show the advantages of working with Dubrovin valuation rings on central simple algebras, even if one were primarily interested in valuation rings in division rings. For, with crossed product constructions and with tensor products, the end result is usually a central simple algebra, and it can be very difficult to get back to the underlying division algebra. It was precisely this difficulty that made some of the proofs in [JW] awkward and indirect.

Our main result here is Theorem 2.1 on extending integral Dubrovin valuation rings to generalized crossed product algebras. This theorem is applied in $\S 3$ to give generalizations to integral Dubrovin valuation rings of the following theorems on valued division algebras over Henselian fields: the inertial extension theorem [JW, Theorem 3.1], the homological description of inertially split division algebras [JW, Theorem 5.6(b)] and the description of the value group

Received by the editors February 22, 1989.

1980 Mathematics Subject Classification (1985 Revision). Primary 13A18, 16A39.

This paper constitutes part of the first author's doctoral dissertation. This author was supported as a research assistant by the N.S.F. during his final year of graduate school.

The second author was partially supported by the N.S.F. 
and residue ring of the tensor product of an inertially split and a totally ramified division algebra [JW, Theorem 6.3]. In addition, we give another tensor product theorem generalizing [ $\mathrm{W}_{2}$, Proposition 2]. This generalization was invoked already in $\left[\mathrm{W}_{2}\right]$ in determining the possible residue rings of division algebras over a Henselian field. Finally, in $\S 4$ we construct a few examples showing the need for some of the hypotheses for the theorems in $\S 3$.

The notation and terminology of this paper will follow $\left[\mathrm{M}_{2}\right]$. In particular, if $B$ is a Dubrovin valuation ring of a central simple $F$-algebra $S$, and if $V=$ $B \cap F$ (a valuation ring of $F$ ), $J(B)$ is the Jacobson radical of $B, \bar{B}=B / J(B)$, $S^{*}$ is the group of units of $S, \operatorname{st}(B)=\left\{s \in S^{*} \mid s B s^{-1}=B\right\}, \Gamma_{B}=s t(B) / B^{*}$, the (linearly ordered) value group of $B$, and $\theta_{B}: \Gamma_{B} / \Gamma_{V} \rightarrow \mathscr{G}(Z(\bar{B}) / \bar{V})$ is the map induced by conjugation by elements of $\operatorname{st}(B)$. If $\left(F_{h}, V_{h}\right)$ is the Henselization of $(F, V)$ then $n_{B}$ is the matrix size of $S \otimes_{F} F_{h}, t_{B}$ is the matrix size of $\bar{B}$ and $s_{B}=n_{B} / t_{B}$ (an integer by $\left[\mathrm{W}_{1}\right.$, Theorem D]).

\section{THE MAIN THEOREM}

In this section we prove the main theorem of this paper. The generality of Theorem 2.1 makes it somewhat cumbersome to state. However, no less general formulation would cover all the applications given in $\S 3$. Since the theorem deals with generalized crossed products, we give a very brief introduction to generalized crossed products.

Recall that a central simple $F$-algebra $S$ is called a crossed product when $S$ contains a subfield $K$ such that $K / F$ is Galois and $[K: F]=\sqrt{[S: F]}=$ $\operatorname{deg}(S)$. In this case $S$ has a direct sum decomposition as a $K$-vector space in which multiplication is described completely by $K$, the Galois group $G=$ $\mathscr{G}(K / F)$, and a certain 2-cocycle in $Z^{2}\left(G, K^{*}\right)$.

A more general situation is for $S$ to contain a Galois extension $K$ of $F$ which may have $[K: F]<\sqrt{[S: F]}$. Such an algebra is then called a generalized crossed product for $K$ over $F$. Generalized crossed products are discussed in $\left[\mathrm{T}, \mathrm{J}_{1}, \mathbf{J}_{2}\right.$ and $\left.\mathrm{KY}\right]$. Just as for crossed products, generalized crossed products can be described "homologically": let $S$ be a central simple $F$-algebra and $K \subseteq S$ a subfield Galois over $F$ with $G=\mathscr{G}(K / F)$. Let $C=C_{S}(K)$, the centralizer of $K$ in $S$. So $C$ is a central simple $K$-algebra and $\operatorname{deg}(S)=\operatorname{deg}(C)[K: F]$. By the Noether-Skolem theorem there are $x_{\sigma} \in S^{*}$ for $\sigma \in G$ such that $x_{\sigma} a x_{\sigma}^{-1}=\sigma(a)$ for all $a \in K$. For $\sigma, \tau \in G, x_{\sigma} x_{\tau} x_{\sigma \tau}^{-1}$ centralizes $K$, so lies in $C^{*}$. Let $\omega: G \rightarrow \operatorname{Aut}(C)$ and $f: G \times G \rightarrow C^{*}$ be defined by

$$
\omega_{\sigma}=\omega(\sigma)=\left.\operatorname{inn}\left(x_{\sigma}\right)\right|_{C}, \quad f(\sigma, \tau)=x_{\sigma} x_{\tau} x_{\sigma \tau}^{-1},
$$

where $\operatorname{inn}(s)$ is the map on $S$ given by $a \mapsto \operatorname{sas}^{-1}$ for $s \in S^{*}$. We then have

(1) $\omega_{\sigma}(a)=\sigma(a)$ for $a \in K$,

(2) $\omega_{\sigma} \omega_{\tau}=\operatorname{inn}(f(\sigma, \tau)) \omega_{\sigma \tau}$ for all $\sigma, \tau \in G$,

(3) $\omega_{\sigma}(f(\tau, \rho)) f(\sigma, \tau \rho)=f(\sigma, \tau) f(\sigma \tau, \rho)$ for all $\sigma, \tau, \rho \in G$. 
Furthermore, $S=\bigoplus_{\sigma \in G} C x_{\sigma}$, and multiplication is given by

$$
\left(c x_{\sigma}\right)\left(d x_{\tau}\right)=\left[c \omega_{\sigma}(d) f(\sigma, \tau)\right] x_{\sigma \tau} .
$$

The couple $(\omega, f)$ satsifying (1)-(3) is called a generalized cocycle. Conversely, starting with a central simple $K$-algebra $C$ and a generalized cocycle $(\omega, f)$ one can construct an algebra $T$ as $T=\bigoplus_{\sigma \in G} C x_{\sigma}$ with multiplication given by (*) (extended bilinearly to $T$ ). It is known that $T$ is a central simple $F$-algebra containing copies of $C$ and $K$ with $C=C_{T}(K)$. This generalized crossed product for $K$ over $F$ is denoted $(C, G,(\omega, f))$. Just as in the case of ordinary cocycles there is a notion of equivalence of generalized cocycles; changing a generalized cocycle to an equivalent one corresponds to changing the choice of the $x_{\sigma}$ (to $c_{\sigma} x_{\sigma}$ where $c_{\sigma} \in C$ ). Furthermore, one calls a generalized cocycle normalized when $x_{\text {id }}=1$, that is when $\omega_{\text {id }}=$ id and $f(\mathrm{id}, \tau)=f(\tau, \mathrm{id})=1$ for all $\tau \in G$. The proofs of these statements are straightforward and can be found in the references given above.

Given a generalized crossed product $S=(C, G,(\omega, f))$ one would like to find out information about Dubrovin valuation rings of $S$ from information known about Dubrovin valuation rings of $C$ and vice versa. The following result does this for integral Dubrovin valuation rings.

For this result we set up some notation. Let $C$ be a central simple $K$-algebra. Suppose $C$ has an integral Dubrovin valuation ring $A$, i.e. $A$ is a Dubrovin valuation ring integral over its center. Let $W=A \cap K$. Suppose $K$ is a Galois extension of a field $F$ with $G=\mathscr{G}(K / F),|G|=n, V=W \cap F$ and that $W / V$ is indecomposed. Let $(\omega, f)$ be a normalized generalized cocycle and $S$ the generalized crossed product $(C, G,(\omega, f))$. Say $S=\bigoplus_{\sigma \in G} C y_{\sigma}$, with multiplication given by $(*)$. We first notice that since $W / V$ is indecomposed, $\sigma(W)=W$ for all $\sigma \in G$, so $y_{\sigma} A y_{\sigma}{ }^{-1}$ is a Dubrovin valuation ring lying over $W$ in $C$, hence by $\left[\mathrm{W}_{1}\right.$, Theorem $\left.\mathrm{A}\right]$ there are $c_{\sigma} \in C^{*}$ with $y_{\sigma} A y_{\sigma}^{-1}=$ $c_{\sigma} A c_{\sigma}^{-1}$. So if $x_{\sigma}=c_{\sigma}^{-1} y_{\sigma}\left(x_{\mathrm{id}}=1\right)$ then $x_{\sigma} A x_{\sigma}^{-1}=A$ and $S=\bigoplus_{\sigma \in G} C x_{\sigma}$. Replacing the $y_{\sigma}$ by the $x_{\sigma}$ corresponds to replacing $(\omega, f)$ by an equivalent cocycle, as mentioned above. So this change does not affect the algebra $S$. We assume $(\omega, f)$ has been modified this way. It was shown in $\left[\mathbf{M}_{2}\right.$, Corollary 2.5] there is a value function $w$ on $C$ corresponding to $A$. Using $w$, we define a function $w^{\prime}$ on $S$ by $w^{\prime}\left(\sum_{\sigma \in G} c_{\sigma} x_{\sigma}\right)=\min _{\sigma}\left\{w\left(c_{\sigma}\right)+w^{\prime}\left(x_{\sigma}\right)\right\}$, where $w^{\prime}\left(x_{\sigma}\right)=\frac{1}{n} \sum_{i=1}^{n} w\left(f\left(\sigma^{i}, \sigma\right)\right)$ in the divisible hull $\Delta_{A}$ of $\Gamma_{A}$. Set $B=\{s \in S \mid$ $\left.w^{\prime}(s) \geq 0\right\}$.

Theorem 2.1. With the notation above, $w^{\prime}$ is a value function on $S$ and the map $\alpha: G \rightarrow \Delta_{A} / \Gamma_{A}$ given by $\alpha(\sigma)=w^{\prime}\left(x_{\sigma}\right)+\Gamma_{A}$ is a homomorphism. Let $I=\operatorname{ker}(\alpha)$. Suppose that the $\omega_{\sigma}$ induce distinct automorphisms of $Z(\bar{A})$ for $\sigma \in I$. Then $B$ is an integral Dubrovin valuation ring with

$$
\Gamma_{B}=\Gamma_{A}+\left\langle\left\{w^{\prime}\left(x_{\sigma}\right) \mid \sigma \in G\right\}\right\rangle,
$$


hence $\Gamma_{B} / \Gamma_{A}=i m(\alpha) \cong G / I$. Furthermore, $\bar{B}$ is a generalized crossed product $\left(\bar{A}, I^{\prime},(\bar{\omega}, g)\right)$, where $I^{\prime}$ is a group isomorphic to $I$ and consists of the automorphisms of $Z(\bar{A})$ induced by the $\omega_{\sigma}$. So $Z(\bar{B})=\mathscr{F}\left(I^{\prime}\right) \subseteq Z(\bar{A})$. In addition, $B$ is a compatible extension of $A, \delta(B)=\delta(A) \delta(W / V), \theta_{B}\left(w^{\prime}\left(x_{\sigma}\right)+\Gamma_{V}\right)=$ $\left.\bar{\omega}_{\sigma}\right|_{Z(\bar{B})}$ for $\sigma \in G$, and the following diagram is commutative:

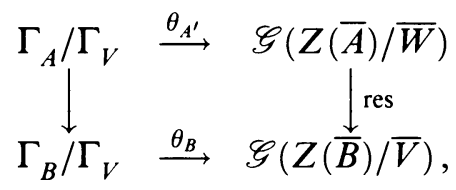

where $\theta_{A^{\prime}}$ is the map induced by $\theta_{A}$.

Proof. Since we are assuming that the $x_{\sigma}$ have been chosen to satisfy $x_{\sigma} A x_{\sigma}^{-1}=$ $A$, it is clear that $w \circ \omega_{\sigma}=w$, hence $f(\sigma, \tau) \in \operatorname{st}(A)$ for all $\sigma, \tau \in G$. The equation

$$
w^{\prime}\left(x_{\sigma}\right)+w^{\prime}\left(x_{\tau}\right)=w(f(\sigma, \tau))+w^{\prime}\left(x_{\sigma \tau}\right)
$$

can be seen as follows: let $c=\operatorname{deg}(C), s=\operatorname{deg}(S)$ and $n=[K: F]$. So $c n=s$. As $x_{\sigma} x_{\tau}=f(\sigma, \tau) x_{\sigma \tau}$, if $v=\left.w\right|_{K}$, a valuation on $K$, we have

$$
v\left(N r d\left(x_{\sigma}\right)\right)+v\left(N r d\left(x_{\tau}\right)\right)=v(N r d(f(\sigma, \tau)))+v\left(N r d\left(x_{\sigma \tau}\right)\right) .
$$

If $N$ is the reduced norm on $C$, then $\left.N r d\right|_{C}=N_{K / F} \circ N$ by [R, 9.14], and since $x_{\sigma}^{n}=\prod_{i=1}^{n} f\left(\sigma^{i}, \sigma\right) \in C$,

$$
\begin{aligned}
v\left(N r d\left(x_{\sigma}\right)\right) & =\frac{1}{n} v\left(N r d\left(x_{\sigma}^{n}\right)\right)=\frac{1}{n} v\left(N_{K / F}\left(N\left(x_{\sigma}^{n}\right)\right)\right) \\
& =v\left(N\left(x_{\sigma}\right)\right)=\sum_{i=1}^{n} v\left(N\left(f\left(\sigma^{i}, \sigma\right)\right)\right)=c \sum_{i=1}^{n} \frac{1}{c} v\left(N\left(f\left(\sigma^{i}, \sigma\right)\right)\right) \\
& =c \sum_{i=1}^{n} w\left(f\left(\sigma^{i}, \sigma\right)\right)=s w^{\prime}\left(x_{\sigma}\right)
\end{aligned}
$$

as $W / V$ is indecomposed and $w(t)=\frac{1}{c} v(N(t))$ for $t \in s t(A)$. Also

$$
\begin{aligned}
v(N r d(f(\sigma, \tau)) & =v\left(N_{K / F}(N(f(\sigma, \tau)))\right. \\
& =n v(N(f(\sigma, \tau)))=\frac{s}{c} v(N(f(\sigma, \tau))=s w(f(\sigma, \tau)) .
\end{aligned}
$$

Using this in (2.3) and dividing by $s$ gives (2.2). This shows $\alpha$ is a homomorphism. We verify that $w^{\prime}$ satisfies the axioms for a value function given in $\left[\mathrm{M}_{2}\right.$, Definition 2.1]:

(1) $w^{\prime}(s+t) \geq \min \left\{w^{\prime}(s), w^{\prime}(t)\right\}$ : Say $s=\sum_{\sigma} c_{\sigma} x_{\sigma}$ and $t=\sum_{\sigma} d_{\sigma} x_{\sigma}$. So $w^{\prime}(s+t)=\min _{\sigma}\left\{w\left(c_{\sigma}+d_{\sigma}\right)+w^{\prime}\left(x_{\sigma}\right)\right\}$. Suppose the minimum occurs at $\tau$ and that $w\left(c_{\tau}\right) \leq w\left(d_{\tau}\right)$. Then

$$
\begin{aligned}
w^{\prime}(s+t) & =w\left(c_{\tau}+d_{\tau}\right)+w^{\prime}\left(x_{\tau}\right) \geq w\left(c_{\tau}\right)+w^{\prime}\left(x_{\tau}\right) \\
& \geq \min _{\sigma}\left\{w\left(c_{\sigma}\right)+w^{\prime}\left(x_{\sigma}\right)\right\}=w^{\prime}(s) \\
& \geq \min \left\{w^{\prime}(s), w^{\prime}(t)\right\} .
\end{aligned}
$$


(2) $w^{\prime}(s t) \geq w^{\prime}(s)+w^{\prime}(t)$ : First, if $s=c_{\sigma} x_{\sigma}$ and $t=d_{\tau} x_{\tau}$ then

$$
\begin{aligned}
w^{\prime}(s t) & =w^{\prime}\left(c_{\sigma} \omega_{\sigma}\left(d_{\tau}\right) f(\sigma, \tau) x_{\sigma \tau}\right)=w\left(c_{\sigma} \omega_{\sigma}\left(d_{\tau}\right) f(\sigma, \tau)\right)+w^{\prime}\left(x_{\sigma \tau}\right) \\
& \geq w\left(c_{\sigma}\right)+w\left(\omega_{\sigma}\left(d_{\tau}\right)\right)+w(f(\sigma, \tau))+w^{\prime}\left(x_{\sigma \tau}\right) \\
& =w\left(c_{\sigma}\right)+w\left(d_{\tau}\right)+w^{\prime}\left(x_{\sigma}\right)+w^{\prime}\left(x_{\tau}\right)=w^{\prime}(s)+w^{\prime}(t)
\end{aligned}
$$

by (2.2). In general, write $s=\sum_{\sigma \in G} s_{\sigma}$ and $t=\sum_{\tau \in G} t_{\tau}$ where $s_{\sigma}=c_{\sigma} x_{\sigma}$ and $t_{\tau}=d_{\tau} x_{\tau}$. Then

$$
\begin{aligned}
w^{\prime}(s t) & =w^{\prime}\left(\sum_{\sigma, \tau} s_{\sigma} t_{\tau}\right) \geq \min _{\sigma, \tau}\left\{w^{\prime}\left(s_{\sigma} t_{\tau}\right)\right\} \\
& \geq \min _{\sigma, \tau}\left\{w^{\prime}\left(s_{\sigma}\right)+w^{\prime}\left(t_{\tau}\right)\right\}=\min _{\sigma}\left\{w^{\prime}\left(s_{\sigma}\right)\right\}+\min _{\tau}\left\{w^{\prime}\left(t_{\tau}\right)\right\} \\
& =w^{\prime}(s)+w^{\prime}(t) .
\end{aligned}
$$

(3) $\operatorname{im}\left(w^{\prime}\right)=w^{\prime}\left(s t\left(w^{\prime}\right)\right)$ : Recall that $s t\left(w^{\prime}\right)=\left\{s \in S^{*} \mid w^{\prime}\left(s^{-1}\right)=\right.$ $\left.-w^{\prime}(s)\right\}$. By the definition of $w^{\prime}, \operatorname{im}\left(w^{\prime}\right)=i m(w)+\left\langle\left\{w^{\prime}\left(x_{\sigma}\right) \mid \sigma \in G\right\}\right\rangle$. Now $\operatorname{im}(w)=w(s t(w)) \subseteq w^{\prime}\left(s t\left(w^{\prime}\right)\right)$ since $\left.w^{\prime}\right|_{C}=w$. Because $x_{\sigma}^{-1}=$ $f\left(\sigma^{-1}, \sigma\right)^{-1} x_{\sigma^{-1}}, w^{\prime}\left(x_{\sigma}^{-1}\right)=-w\left(f\left(\sigma^{-1}, \sigma\right)\right)+w^{\prime}\left(x_{\sigma^{-1}}\right)$. Also, by (2.2), since $x_{\sigma^{-1}} x_{\sigma}=f\left(\sigma^{-1}, \sigma\right), w^{\prime}\left(x_{\sigma^{-1}}\right)+w^{\prime}\left(x_{\sigma}\right)=w\left(f\left(\sigma^{-1}, \sigma\right)\right)$. Thus $w^{\prime}\left(x_{\sigma}^{-1}\right)=$ $-w\left(x_{\sigma}\right)$, so $x_{\sigma} \in s t\left(w^{\prime}\right)$. Therefore $\operatorname{im}\left(w^{\prime}\right) \subseteq w^{\prime}\left(s t\left(w^{\prime}\right)\right)$, hence $\operatorname{im}\left(w^{\prime}\right)=$ $w^{\prime}\left(s t\left(w^{\prime}\right)\right)$. Thus $w^{\prime}$ is a value function on $S$.

Let $B=\left\{s \in S \mid w^{\prime}(s) \geq 0\right\}$ and $J=\left\{s \in S \mid w^{\prime}(s)>0\right\}$. To prove $B$ is a Dubrovin valuation ring, by $\left[\mathrm{M}_{2}\right.$, Theorem 2.4] we need to see that $B / J$ is simple Artinian. We will show $B / J$ is a generalized crossed product over $\bar{A}$. Now $I=\operatorname{ker}(\alpha)=\left\{\sigma \in G \mid w^{\prime}\left(x_{\sigma}\right) \in \Gamma_{A}\right\}$. So, for $\sigma \in I$ there is an $a_{\sigma} \in s t(A) \subseteq C^{*}$ with $w^{\prime}\left(x_{\sigma}\right)=w^{\prime}\left(a_{\sigma}\right)$. We now modify the $x_{\sigma}$ (and modify $(\omega, f)$ to an equivalent generalized cocycle) by replacing $x_{\sigma}$ by $a_{\sigma}^{-1} x_{\sigma}$ for $\sigma \in I$. With the new $x_{\sigma}$ we have $w^{\prime}\left(x_{\sigma}\right)=0$ for $\sigma \in I$. Thus $w(f(\sigma, \tau))=0$ for $\sigma, \tau \in I$, by (2.2). As $A^{*}=s t(A) \cap(A-J(A))$ by [W ${ }_{1}$, Lemma 3.3], $f(\sigma, \tau) \in A^{*}$ for $\sigma, \tau \in I$.

Let $\bar{\omega}_{\sigma}$ be the automorphism of $\bar{A}$ induced by conjugation by $x_{\sigma}$, and $\psi_{\sigma}=\left.\bar{\omega}_{\sigma}\right|_{Z(\bar{A})}$. The $\psi_{\sigma}$ are distinct (for $\sigma \in I$ ) by assumption. Let $I^{\prime}=$ $\left\{\psi_{\sigma} \mid \sigma \in I\right\}$ and $g\left(\psi_{\sigma}, \psi_{\tau}\right)=\overline{f(\sigma, \tau)} \in \bar{A}^{*}$ for $\sigma, \tau \in I$. We show $I^{\prime}$ is a group and $(\bar{\omega}, g)$ is a generalized cocycle for $Z(\bar{A}) / \mathscr{F}\left(I^{\prime}\right)$. First, to show $\bar{\omega}_{\sigma} \bar{\omega}_{\tau}=\operatorname{inn}\left(g\left(\psi_{\sigma}, \psi_{\tau}\right)\right) \bar{\omega}_{\sigma \tau}$, for $a \in A$,

$$
\begin{aligned}
\bar{\omega}_{\sigma} \bar{\omega}_{\tau}(\bar{a}) & =\bar{\omega}_{\sigma}\left(\overline{x_{\tau} a x_{\tau}^{-1}}\right)=\overline{x_{\sigma} x_{\tau} a x_{\tau}^{-1} x_{\sigma}^{-1}}=\overline{\omega_{\sigma} \omega_{\tau}(a)} \\
& =\overline{f(\sigma, \tau) \omega_{\sigma \tau}(a) f(\sigma, \tau)^{-1}}=\overline{f(\sigma, \tau)} \overline{\omega_{\sigma \tau}(a)} \overline{f(\sigma, \tau)} \\
& =g\left(\psi_{\sigma}, \psi_{\tau}\right) \bar{\omega}_{\sigma \tau}(\bar{a}) g\left(\psi_{\sigma}, \psi_{\tau}\right)^{-1}=\left(\operatorname{inn}\left(g\left(\psi_{\sigma}, \psi_{\tau}\right)\right) \bar{\omega}_{\sigma \tau}\right)(\bar{a})
\end{aligned}
$$

as $(\omega, f)$ is a generalized cocycle. Furthermore, if $\bar{a} \in Z(\bar{A}), \psi_{\sigma \tau}(\bar{a})=$ $\bar{\omega}_{\sigma \tau}(\bar{a}) \in Z(\bar{A})$, so $g\left(\psi_{\sigma}, \psi_{\tau}\right) \bar{\omega}_{\sigma \tau}(\bar{a}) g\left(\psi_{\sigma}, \psi_{\tau}\right)^{-1}=\bar{\omega}_{\sigma \tau}(\bar{a})$. Therefore $\psi_{\sigma} \psi_{\tau}=$ 
$\psi_{\sigma \tau}$. This shows $I^{\prime}$ is a group and the map $I \rightarrow I^{\prime}$ given by $\sigma \mapsto \psi_{\sigma}$ is a homomorphism. Since the $\psi_{\sigma}$ are distinct, $I \cong I^{\prime}$. Second, we need to show $\bar{\omega}_{\sigma}\left(g\left(\psi_{\tau}, \psi_{\rho}\right)\right) g\left(\psi_{\sigma}, \psi_{\tau \rho}\right)=g\left(\psi_{\sigma}, \psi_{\tau}\right) g\left(\psi_{\sigma \tau}, \psi_{\rho}\right)$. To see this,

$$
\begin{aligned}
\bar{\omega}_{\sigma}\left(g\left(\psi_{\tau}, \psi_{\rho}\right)\right) g\left(\psi_{\sigma}, \psi_{\tau \rho}\right) & =\bar{\omega}_{\sigma}(\overline{f(\tau, \rho)}) \overline{f(\sigma, \tau \rho)}=\overline{\omega_{\sigma}(f(\tau, \rho)) f(\sigma, \tau \rho)} \\
& =\overline{f(\sigma, \tau)} \overline{f(\sigma \tau, \rho)}=g\left(\psi_{\sigma}, \psi_{\tau}\right) g\left(\psi_{\sigma \tau}, \psi_{\rho}\right) .
\end{aligned}
$$

Thus $(\bar{\omega}, g)$ is a generalized cocycle; let $T$ be the central simple algebra $\left(\bar{A}, I^{\prime},(\bar{\omega}, g)\right)$. We identify $I^{\prime}$ with $I$ when used as index sets. Thus, we write $T=\bigoplus_{\sigma \in I} \bar{A} y_{\sigma}$ with $y_{\sigma} \bar{a} y_{\sigma}^{-1}=\bar{\omega}_{\sigma}(\bar{a})$ for $\bar{a} \in \bar{A}$ and $y_{\sigma} y_{\tau} y_{\sigma \tau}^{-1}=g\left(\psi_{\sigma}, \psi_{\tau}\right)$. If $\sum_{\sigma \in G} c_{\sigma} x_{\sigma} \in B$ then $w\left(c_{\sigma}\right)+w^{\prime}\left(x_{\sigma}\right) \geq 0$ for all $\sigma \in G$. Hence $c_{\sigma} \in A$ for $\sigma \in I$ since $w^{\prime}\left(x_{\sigma}\right)=0$. For $\sigma \notin I, w^{\prime}\left(x_{\sigma}\right) \notin \Gamma_{A}$, so $w\left(c_{\sigma}\right)+w^{\prime}\left(x_{\sigma}\right)>0$. Define $h: B \rightarrow T$ by $h\left(\sum_{\sigma \in G} c_{\sigma} x_{\sigma}\right)=\sum_{\sigma \in I} \bar{c}_{\sigma} y_{\sigma}$. Clearly $h$ is an additive group homomorphism, and $\operatorname{ker}(h)=\left\{\sum_{\sigma \in G} c_{\sigma} x_{\sigma} \in B \mid c_{\sigma} \in J(A)\right.$ for all $\sigma \in$ $I\}=\left\{\sum_{\sigma \in G} c_{\sigma} x_{\sigma} \in S \mid w^{\prime}\left(\sum_{\sigma \in G} c_{\sigma} x_{\sigma}\right)>0\right\}=J$. Surjectivity of $h$ is also clear. To see $h$ preserves multiplication, if $\sum_{\sigma \in G} c_{\sigma} x_{\sigma}$ and $\sum_{\tau \in G} d_{\tau} x_{\tau} \in B$,

$$
\sum_{\sigma \in G} c_{\sigma} x_{\sigma} \cdot \sum_{\tau \in G} d_{\tau} x_{\tau}=\sum_{\rho \in G}\left(\sum_{\sigma \tau=\rho} c_{\sigma} \omega_{\sigma}\left(d_{\tau}\right) f(\sigma, \tau)\right) x_{\rho} .
$$

If $\rho \in I$ and $\sigma \tau=\rho$ with $\sigma \notin I$ then $w^{\prime}\left(c_{\sigma} x_{\sigma}\right)$ and $w^{\prime}\left(d_{\tau} x_{\tau}\right)>0$; so $w\left(c_{\sigma} \omega_{\sigma}\left(d_{\tau}\right) f(\sigma, \tau)\right)=w^{\prime}\left(\left(c_{\sigma} x_{\sigma}\right)\left(d_{\tau} x_{\tau}\right)\right) \geq w^{\prime}\left(c_{\sigma} x_{\sigma}\right)+w^{\prime}\left(d_{\tau} x_{\tau}\right)>0$. So

$$
\begin{aligned}
h\left(\sum_{\sigma \in G} c_{\sigma} x_{\sigma} \cdot \sum_{\tau \in G} d_{\tau} x_{\tau}\right) & =\sum_{\rho \in I} \sum_{\sigma \tau=\rho} \overline{c_{\sigma} \omega_{\sigma}\left(d_{\tau}\right) f(\sigma, \tau)} y_{\rho} \\
& =\sum_{\sigma, \tau \in I} \overline{c_{\sigma}} \bar{\omega}_{\sigma}\left(\overline{d_{\tau}}\right) g\left(\psi_{\sigma}, \psi_{\tau}\right) y_{\sigma \tau}=\sum_{\sigma \in I} \overline{c_{\sigma}} y_{\sigma} \cdot \sum_{\tau \in I} \overline{d_{\tau}} y_{\tau} .
\end{aligned}
$$

Hence $h$ is a ring homomorphism, and so $B / J \cong T$. Thus by $\left[\mathrm{M}_{2}\right.$, Theorem 2.4], $B$ is a Dubrovin valuation ring of $S$. From the construction of $w^{\prime}$ it is clear that $\Gamma_{B}=i m\left(w^{\prime}\right)=\Gamma_{A}+\left\langle\left\{w^{\prime}\left(x_{\sigma}\right)\right\}\right\rangle$, so $\Gamma_{B} / \Gamma_{A}=\operatorname{im}(\alpha) \cong G / I$ and $\bar{B}=B / J=\left(\bar{A}, I^{\prime},(\bar{\omega}, g)\right)$. Since $\left.w^{\prime}\right|_{C}=w, J(A)=J \cap A$ and $\operatorname{st}(A)=$ $s t(w) \subseteq s t\left(w^{\prime}\right)=\operatorname{st}(B)$. Hence $B / A$ is compatible. The commutativity of the diagram is clear. By definition of $\theta_{B}$ and the isomorphism $\Gamma_{B} / \Gamma_{V} \cong \operatorname{im}\left(w^{\prime}\right)$, since $x_{\sigma} \in \operatorname{st}(B), \theta_{B}\left(w^{\prime}\left(x_{\sigma}\right)+\Gamma_{V}\right)$ is the map on $Z(\bar{B})$ induced by conjugation by $x_{\sigma}$, so $\theta_{B}\left(w^{\prime}\left(x_{\sigma}\right)+\Gamma_{V}\right)=\left.\bar{\omega}_{\sigma}\right|_{Z(\bar{B})}=\psi_{\sigma}$. Finally, for the information about the defect, we have

$$
\begin{aligned}
{[S: F] } & =|G|[C: K][K: F] \\
& =|G / I||I|\left|\Gamma_{A}: \Gamma_{W}\right|[\bar{A}: \bar{W}] \delta(A)\left|\Gamma_{W}: \Gamma_{V}\right|[\bar{W}: \bar{V}] \delta(W / V) \\
& =\left(\left|\Gamma_{B}: \Gamma_{A}\right|\left|\Gamma_{A}: \Gamma_{W}\right|\left|\Gamma_{W}: \Gamma_{V}\right|\right)(|I|[\bar{A}: \bar{W}][\bar{W}: \bar{V}]) \delta(A) \delta(W / V) \\
& =\left|\Gamma_{B}: \Gamma_{V}\right|[\bar{B}: \bar{V}] \delta(A) \delta(W / V) .
\end{aligned}
$$

So, $\delta(B)=\delta(A) \delta(W / V)$. 
An alternative way to define $w^{\prime}$ above is to set $w^{\prime}\left(x_{\sigma}\right)=\frac{1}{s} w\left(N r d\left(x_{\sigma}\right)\right)$. It is shown in the proof of Theorem 2.1 that $x_{\sigma} \in s t(B)$, so by $\left[\mathrm{M}_{2}\right.$, Proposition 2.6] this formula for $w^{\prime}\left(x_{\sigma}\right)$ is valid. While this definition would simplify the proof somewhat, it does not lend itself as well to actual computations.

Corollary 2.4. With notation of Theorem 2.1, let $H$ be the kernel of the natural homomorphism $\mathscr{G}(K / F) \rightarrow \mathscr{G}(\bar{W} / \bar{V})$. Suppose $I \cap H=0$. Then for $\sigma \in I$ the $\omega_{\sigma}$ induce distinct automorphisms of $Z(\bar{A})$, hence the conclusions of Theorem 2.1 apply.

Proof. The assumption that $I \cap H=0$ means that the $\sigma \in I$ induce distinct automorphisms of $\bar{W}$. Since $\bar{W} \subseteq Z(\bar{A})$, this assures that the $\omega_{\sigma}$ induce distinct automorphisms of $Z(\bar{A})$, which is what is needed for Theorem 2.1.

\section{Applications of the MAIN theOREM}

In this section we will make use of Theorem 2.1 to generalize results of [JW and $\mathrm{W}_{2}$ ] to integral Dubrovin valuation rings. The first consequence is a generalization of [JW, Theorem 3.1] for Galois inertial extensions. We will follow the notation of $\S 2$. Note that if $K / F$ is inertial (= unramified) with respect to $W / V$ then the $H$ of Corollary 2.4 is zero, so the condition $I \cap H=0$ is automatically satisfied. This is the case here.

Theorem 3.1. Suppose $S$ is a central simple $F$-algebra, $K / F$ an inertial Galois extension relative to the valuation rings $W / V$. Let $A$ be a Dubrovin valuation ring in $S \otimes_{F} K$ with $A \cap K=W$, and $B$ a Dubrovin valuation ring in $S$ with $B \cap F=V$. Then, $Z(\bar{A})=Z(\bar{B}) \bar{W}, \bar{A} \sim \bar{B} \otimes_{Z(\bar{B})} Z(\bar{B}) \bar{W}, \Gamma_{A} / \Gamma_{W}=$ $\theta_{B}^{-1}(\mathscr{G}(Z(\bar{B}) /(Z(\bar{B}) \cap \bar{W}))), \delta(A)=\delta(B)$ and $M_{n}(\bar{B})$ is a generalized crossed product over $\bar{A}$, where $n=[K: F]$. Furthermore, $s_{B} \leq s_{A}$. In particular, if $A$ is integral then $B$ is integral.

Proof. First suppose $A$ is integral. We have $K \cong F \otimes_{F} K \subseteq S \otimes_{F} M_{n}(F)=$ $M_{n}(S)$ and $S \otimes_{F} K=C_{M_{n}(S)}\left(F \otimes_{F} K\right)$. So $M_{n}(S)$ is a generalized crossed product over $S \otimes_{F} K$ with group $G$. Thus by Corollary 2.4, $M_{n}(S)$ contains an integral Dubrovin extension $B_{0}$ of $V$. Hence by [D $\mathrm{D}_{1}$, Theorem 7, p. 280], $S$ contains a Dubrovin valuation ring $B_{1} / V$ and $B_{0} \cong M_{n}\left(B_{1}\right) . B_{1}$ is integral as $B_{0}$ is integral. By $\left[\mathrm{W}_{1}\right.$, Theorem $\left.\mathrm{A}\right], B_{1} \cong B$. Since $\overline{B_{0}} \cong M_{n}(\bar{B})$ is a generalized crossed product over $\bar{A}, \bar{A}=C_{\overline{B_{0}}}(Z(\bar{A}))$; hence $\bar{A} \sim \overline{B_{0}} \otimes_{Z\left(\overline{B_{0}}\right)}$ $Z(\bar{A}) \sim \bar{B} \otimes_{Z(\bar{B})} Z(\bar{A}) . Z(\bar{A}) / Z(\bar{B})$ is a Galois extension with group $I^{\prime}$, and no element of $I^{\prime}$ other than the identity fixes $\bar{W}$ since $\left.\psi_{\sigma}\right|_{\bar{W}}=\bar{\sigma} \neq$ id for $\sigma \neq \mathrm{id}$. Hence $\mathscr{G}(Z(\bar{A}) / Z(\bar{B}) \bar{W})=\{i d\}$, so $Z(\bar{B}) \bar{W}=Z(\bar{A})$. From the diagram in 2.1, since $\theta_{A}$ and $\theta_{B}$ are surjective we see that the image of $\Gamma_{A} / \Gamma_{W}$ in $\mathscr{G}(Z(\bar{B}) / \bar{V})$ is $\mathscr{G}(Z(\bar{B}) /(Z(\bar{B}) \cap \bar{W}))$, so $\Gamma_{A} / \Gamma_{W}=\theta_{B}^{-1}(\mathscr{G}(Z(\bar{B}) /(Z(\bar{B}) \cap \bar{W})))$. Finally, since $W / V$ is inertial, $\delta(W / V)=1$, so $\delta(A)=\delta\left(B_{0}\right)=\delta(B)$. 
Now drop the assumption of integrality. Let $\left(F_{h}, V_{h}\right)$ and $\left(K_{h}, W_{h}\right)$ be the Henselizations of $(F, V)$ and $(K, W)$ respectively. Let $D$ and $E$ be the underlying division algebras of $S \otimes_{F} F_{h}$ and $\left(S \otimes_{F} K\right) \otimes_{K} K_{h}=S \otimes_{F} K_{h}$. Since $F_{h}$ and $K_{h}$ are Henselian there are invariant valuation rings $R \subseteq D$ and $R^{\prime} \subseteq E$ lying over $V_{h}, W_{h}$. By $\left[\mathrm{W}_{1}\right.$, Theorem $\left.\mathrm{B}\right], \bar{B}=\bar{R}, \bar{A}=\overline{R^{\prime}}, \Gamma_{B}=$ $\Gamma_{R}, \Gamma_{A}=\Gamma_{R^{\prime}}, \delta(B)=\delta(R), \delta(A)=\delta\left(R^{\prime}\right), \theta_{B}=\theta_{R}$ and $\theta_{A}=\theta_{R^{\prime}}$. Therefore applying what has been proven above to $R$ and $M_{r}\left(R^{\prime}\right)$, where $r$ is the matrix size of $D \otimes_{F_{h}} K_{h}$, show the conclusions proven for $A$ integral above hold in general.

To see $s_{B} \leq s_{A}$, let $V \subseteq V_{1} \subseteq V_{2}$ be overrings of $V$ in $F$ and set $B_{i}=B V_{i}$, $W_{i}=W V_{i}$ and $A_{i}=A W_{i}=A V_{i}$. Since $W / V$ is inertial, $W_{i} / V_{i}$ is inertial. The above arguments applied to $B_{2}$ and $A_{2}$ show $Z\left(\overline{B_{2}}\right) \subseteq Z\left(\overline{A_{2}}\right)$. Let $\widetilde{V_{1}}=$ $V_{1} / J\left(V_{2}\right)$ and $\widetilde{W}_{1}=W_{1} / J\left(W_{2}\right)$. So $\widetilde{W}_{1} / \widetilde{V}_{1}$ is also inertial. Let $l_{i}$ (resp. $l_{i}^{\prime}$ ) be the number of extensions of $\widetilde{V}_{1}$ (resp. $\left.\widetilde{W}_{1}\right)$ to $Z\left(\overline{B_{2}}\right)$ (resp. $Z\left(\overline{A_{2}}\right)$ ). Since $\widetilde{W}_{1} / \widetilde{V}_{1}$ is indecomposed, $l_{i}$ is the number of extensions of $\widetilde{W}_{1}$ to $Z\left(\overline{A_{2}}\right)$. As $Z\left(\overline{B_{2}}\right) \subseteq Z\left(\overline{A_{2}}\right), \widetilde{W}_{1}$ extends in at most $l_{i}$ ways to $Z\left(\overline{B_{2}}\right)$. Therefore $l_{i}^{\prime} \leq l_{i}$. Applying this to $\left[\mathrm{W}_{1}\right.$, Theorem $\left.\mathrm{D}\right]$ the $l_{i}$ for $B$ are less than or equal to the corresponding $l_{i}^{\prime}$ for $A$, so $s_{B} \leq s_{A}$.

The following corollary was pointed out to us by Tignol.

Corollary 3.2. Suppose $S$ is a central simple $F$-algebra and $V$ is a valuation ring of $F$. If $S$ is split by a Galois extension $K$ of $F$ containing an inertial extension of $V$, then every Dubrovin valuation ring $B$ of $S$ lying over $V$ is integral over $V$.

Proof. Say $S \otimes_{F} K \cong M_{k}(K)$. If $W$ is the inertial extension of $V$ to $K$, then $M_{k}(W)$ is a Dubrovin valuation ring of $M_{k}(K)$ integral over $W$. Theorem 3.1 shows $B$ is integral over $V$.

In Theorem 3.1 we related the Dubrovin valuation rings $B$ of $S$ and $A$ of $S \otimes_{F} K$ by showing $M_{n}(B)$ is a compatible extension of $A$ (after modifying the choice of $B$ ). A more direct approach would be to choose $A$ and $B$ so that $A$ is a compatible extension of $B$. However, it turns out that this is often not possible. We will show in Proposition 3.4 exactly when this can be done.

Lemma 3.3. Let $S \subseteq T$ be central simple algebras. Then there is a value function $w$ on $T$ (with $B_{w}$ a Dubrovin valuation ring) such that $\left.w\right|_{S}$ is a value function (with $B_{\left.w\right|_{s}}$ a Dubrovin valuation ring) iff there is a compatible extension $A / B$ of integral Dubrovin valuation rings with $B$ a Dubrovin valuation ring of $S$ and $A$ of $T$.

Proof. Suppose $w$ is a value function on $T$ with $\left.w\right|_{S}$ a value function on $S$. If $A$ is the integral Dubrovin valuation ring of $T$ corresponding to $w$ and $B$ corresponding to $\left.w\right|_{S}$, then it is clear that $A / B$ is a compatible extension. Conversely, suppose $A / B$ is compatible. Let $w$ be the value function on $T$ 
corresponding to $A$. Then $B=A \cap S=\{s \in S \mid w(s) \geq 0\}$ and $J(B)=$ $\{s \in S \mid w(s)>0\}$. So if $v=\left.w\right|_{S}$ then $B_{v}=B$ and $J_{v}=J(B)$ where $B_{v}$ and $J_{v}$ are as in $\left[\mathrm{M}_{2}\right.$, Lemma 2.2]. Therefore $B_{v} / J_{v}=\bar{B}$ is simple Artinian. To show $v$ is a value function on $S$ and $B$ is a Dubrovin valuation ring, by $\left[\mathrm{M}_{2}\right.$, Theorem 2.4] it remains to show $\operatorname{im}(v)=v(s t(v))$. Take $s \in S$. Since $B$ is integral, $B s B=\beta B$ for some $\beta \in \operatorname{st}(B)$. So $A s A=\beta A$, therefore $v(s)=w(s)=w(\beta) . w\left(\beta^{-1}\right)=-w(\beta)$ as $\beta \in \operatorname{st}(B) \subseteq \operatorname{st}(A)=s t(w)$. Thus $\beta \in \operatorname{st}(v)$, and $v(s)=w(\beta)=v(\beta) \in(s t(v))$. Therefore $v$ is a value function on $S$.

Proposition 3.4. Let $S, K, F, W, V$ be as in Theorem 3.1, and set $T=$ $S \otimes_{F} K$. Suppose $A$ is integral Dubrovin in $T$ with $A \cap K=W$ and $B$ integral Dubrovin in $S$ with $B \cap F=V$. Then we can choose $A$ so that $A / B$ is compatible iff the following equivalent conditions hold:

(1) $Z(\bar{B})$ and $\bar{W}$ are linearly disjoint over $\bar{V}$,

(2) $Z(\bar{B}) \cap \bar{W}=\bar{V}$,

(3) $\Gamma_{A}=\Gamma_{B}$,

(4) $A \cong B \otimes_{V} W$.

Proof. By Theorem 3.1, $\Gamma_{A}=\theta_{B}^{-1}(\mathscr{G}(Z(\bar{B}) /(Z(\bar{B}) \cap \bar{W}))) \subseteq \Gamma_{B}$. Thus $\Gamma_{A}=\Gamma_{B}$ iff $Z(\bar{B}) \cap \bar{W}=\bar{V}$ iff $Z(\bar{B})$ and $\bar{W}$ are linearly disjoint over $\bar{V}$, as $Z(\bar{B}) / \bar{V}$ is a normal extension and $\bar{W} / \bar{V}$ is separable. When these happen, $\bar{B} \otimes_{\bar{V}} \bar{W}$ is simple, so $B \otimes_{V} W$ is Dubrovin by $\left[\mathrm{M}_{2}\right.$, Theorems 3.3 and 3.4], and is a compatible extension of $B$. Conversely, if $A^{\prime}=B \otimes_{V} W$ is Dubrovin, let $I=J(B) \otimes_{V} W$. Then, $I=J(B) \otimes_{V} W+B \otimes_{V} J(W)$ since $J(W)=J(V) W$. Also, $I \subseteq A^{\prime}$ as $W$ is a flat $V$-module. Then

$$
A^{\prime} / I \cong \bar{B} \otimes_{\bar{V}} \bar{W}=\bar{B} \otimes_{Z(\bar{B})}\left(Z(\bar{B}) \otimes_{\bar{V}} \bar{W}\right) .
$$

Now $Z(\bar{B}) \otimes \bar{V} \bar{W}$ is a direct sum of fields, so $A^{\prime} / I$ is semisimple. As $I \subseteq J\left(A^{\prime}\right)$ (since $I \neq A^{\prime}$ ) this implies that $I=J\left(A^{\prime}\right)$, so $A^{\prime} / I$ is actually simple, hence $Z(\bar{B}) \otimes_{\bar{V}} \bar{W}$ is a field. Thus $Z(\bar{B})$ and $\bar{W}$ are linearly disjoint over $\bar{V}$. On the other hand, if $A / B$ is compatible then [W ${ }_{1}, \S 3$ ] (or Lemma 3.3) shows $\Gamma_{B} \subseteq \Gamma_{A}$. Since $\Gamma_{A} \subseteq \Gamma_{B}$ we get $\Gamma_{A}=\Gamma_{B}$.

In [JW, Theorem 3.1] Jacob and the second author prove a theorem about inertial scalar extensions of Henselian valued fields. By using Dubrovin valuation rings we now give a more natural proof of [JW, Theorem 3.1] in the case of a Galois inertial extension.

Corollary 3.5. Let $(F, V)$ be a Henselian valued field, $(K, W)$ an inertial Galois extension of $(F, V), D$ an $F$-central division algebra and $E$ the underlying division algebra of $D \otimes_{F} K$. If $V_{D}, V_{E}$ are the invariant valuation rings of $D$, $E$ lying over $V, W$ respectively then

(1) $Z\left(\overline{V_{E}}\right)=Z\left(\overline{V_{D}}\right) \bar{W}$.

(2) $\overline{V_{E}} \sim \overline{V_{D}} \otimes_{Z\left(\overline{V_{D}}\right)} Z\left(\overline{V_{D}}\right) \bar{W}$. 
(3) $\Gamma_{V_{E}}=\theta_{V_{D}}^{-1}\left(\mathscr{G}\left(Z\left(\overline{V_{D}}\right) / Z\left(\overline{V_{D}}\right) \cap \bar{W}\right)\right)$.

(4) $\delta\left(V_{E}\right)=\delta\left(V_{D}\right)$.

(5) The following diagram is commutative:

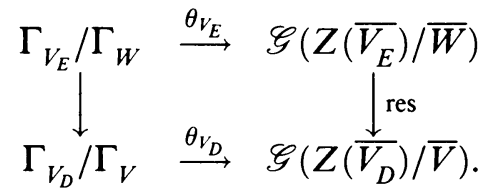

Proof. Since $F$ is Henselian, every central simple algebra $T$ with $F \subseteq Z(T)$ and $[T: F]<\infty$ contains an integral Dubrovin valuation ring lying over the (Henselian) valuation ring $V$ of $F$. As $\Gamma_{M_{n}(B)}=\Gamma_{B}, \overline{M_{n}(B)}=\bar{B}, \delta\left(M_{n}(B)\right)$ $=\delta(B)$ and $\theta_{M_{n}(B)}=\theta_{B}$ for any Dubrovin valuation ring $B$, by [ $\mathrm{W}_{1}$, Corollary 3.6, Theorems $B$ and $C$ ], this corollary follows from Theorem 3.1 and the diagram of Theorem 2.1 .

The next two results deal with inertially split algebras, that is central simple $F$-algebras $S$ such that $S$ has a splitting field $K$ which is an inertial extension of $F$. In [JW, §5] such algebras are described homologically (for $F$ Henselian). Theorem 2.1 allows these results to be seen via Dubrovin valuation rings and generalized.

Theorem 3.6. Let $K / F$ be an inertial Galois extension relative to the valuation rings $W / V$ and let $G=\mathscr{G}(K / F)$. Let $S=(K, G, f)$ be a crossed product algebra for some $f \in Z^{2}\left(G, K^{*}\right)$. Define $\alpha$ and $w^{\prime}$ as in Theorem 2.1, (with $A=W)$. If $B$ is the integral Dubrovin valuation ring of $S$ associated to $w^{\prime}$ then $\Gamma_{B} / \Gamma_{V}=i m(\alpha), \bar{B}=(\bar{W}, \bar{I}, g)$ for some $g \in Z^{2}\left(\bar{I}, \bar{W}^{*}\right)$, where $I=\operatorname{ker}(\alpha)$ and $\bar{I}$ is the image of $I$ in $\mathscr{G}(\bar{W} / \bar{V})$, and $B$ is defectless. Furthermore, if $\alpha_{0}$ is the map $\mathscr{G}(Z(\bar{B}) / \bar{V}) \rightarrow \mathscr{G}(\bar{W} / \bar{V}) \rightarrow \Gamma_{B} / \Gamma_{V}$ induced by $\alpha$, then $\theta_{B}=\alpha_{0}^{-1}$.

Proof. Here $C=K$ and so $B$ is defectless since $W$ is. Everything follows immediately from Theorem 2.1 .

An easy calculation shows that the formula for $\alpha$ is the same as given in [JW, Remark 5.5]. Hence we get a new proof of Theorem 5.6(b) in [JW]:

Corollary 3.7. Let $(F, V)$ be a Henselian valued field and $D$ an inertially split division algebra with $F=Z(D)$. Write $D \sim(K, G, f)$ for any splitting field $K$ of $D$ with $K$ inertial Galois over $F$, and let $\alpha: G \rightarrow \Delta_{V} / \Gamma_{V}$ be the map of Theorem 2.1. If $V_{D}$ is the invariant valuation ring of $D$ lying over $V$, then $V_{D} / V$ is defectless, $\Gamma_{V_{D}} / \Gamma_{V}=i m(\alpha)$ and $Z\left(\overline{V_{D}}\right)=\overline{\mathscr{F}}(\operatorname{ker}(\alpha))$.

Proof. Since $D$ is inertially split and $F$ is Henselian, $D \sim(K, G, f)$ for some $K / F$ inertial Galois. The corollary then follows from Theorem 3.6.

In $[\mathrm{JW}, \S 6]$ it is shown that a tame division algebra $D$ over a Henselian field can be decomposed up to Brauer equivalence into an inertially split division algebra tensored with a totally ramified division algebra. Then information on the value group and the center of the residue division algebra for the valuation 
ring of $D$ is obtained from the tensor factors. We now generalize this to integral Dubrovin valuation rings.

First, to set up notation, let $(F, V)$ be a valued field, $K, L$ Galois extensions of $F$ with $G=\mathscr{G}(L / F), H=\mathscr{G}(K / F), n=|G|$ and $m=|H|$ such that $V$ extends to $V_{K} \subseteq K$ and $V_{L} \subseteq L$ with $V_{K} / V$ tame totally ramified and $V_{L} / V$ inertial. Take $f \in Z^{2}\left(G, L^{*}\right), g \in Z^{2}\left(H, K^{*}\right), S=(L, G, f), T=(K, H, g)$ and suppose $T$ is a totally ramified division algebra with respect to the extension of $v$ to $T$ with invariant valuation ring $B_{T} . S$ has an integral Dubrovin valuation ring $B_{S}$ by Theorem 3.6.

Theorem 3.8. With $S$ and $T$ as above, $S \otimes_{F} T$ contains an integral Dubrovin valuation ring $B$ such that $B$ is defectless, $\Gamma_{B}=\Gamma_{B_{S}}+\Gamma_{B_{T}}, \operatorname{ker}\left(\theta_{B}\right)=\Gamma_{B_{T}}$, $\bar{B}=\left(\overline{V_{L}}, G_{0}, h\right)$ for some $G_{0} \subseteq G$, and $Z(\bar{B})=\mathscr{F}\left(\theta_{B_{S}}\left(\Gamma_{B_{S}} \cap \Gamma_{B_{T}}\right) / \Gamma_{V}\right) \subseteq$ $Z\left(\overline{B_{S}}\right)$. Furthermore, the following diagram commutes:

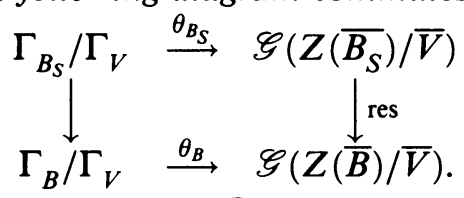

Proof. Write $S=\bigoplus_{\sigma \in G} L x_{\sigma}, T=\bigoplus_{\tau \in H} K y_{\tau}$ where the $x_{\sigma}, y_{\tau}$ have the usual crossed product properties. We assume $f$ and $g$ have been normalized and that $f$ has also been modified to suppose $f(\sigma, \tau) \in \operatorname{st}\left(B_{S}\right)$ as in Theorem 2.1. Let $w$ be the value function on $S$ as in Theorem 2.1 and for convenience also write $w$ for the valuation on $T$. Since $y_{\tau}^{m}=\prod_{i=1}^{m} g\left(\tau^{i}, \tau\right), w\left(y_{\tau}\right)=$ $\frac{1}{m} \sum_{i=1}^{m} w\left(g\left(\tau^{i}, \tau\right)\right)$. Furthermore, the $w\left(y_{\tau}\right)$ are distinct $\bmod \Gamma_{V_{K}}$, and so $w\left(\sum_{\tau \in H} k_{\tau} y_{\tau}\right)=\min _{\tau}\left\{w\left(k_{\tau}\right)+w\left(y_{\tau}\right)\right\}$. To see this, if $w\left(y_{\tau}\right) \in \Gamma_{V_{K}}$, we can modify $y_{\tau}$ to assume $w\left(y_{\tau}\right)=0$. Thus for all $a \in V_{K}, \overline{\tau(a)}=\overline{y_{\tau} a y_{\tau}^{-1}}=$ $\overline{y_{\tau}} \bar{a} \bar{y}_{\tau}^{-1}=\bar{a}$ as $\overline{B_{T}}=\bar{V}$, a field. So $\overline{\tau(a) / a}=1$ for all $a \in K^{*}$. But the proof of [JW, Proposition 2.2] yields $\tau=1$. Let $M=L K$. Since $L \cap K$ is both totally ramified and inertial over $F, L \cap K=F$. Thus $M / F$ is Galois with Galois group $G \times H$. Furthermore, $M / F$ is indecomposed with respect to $V$, and the extension $V_{M}$ of $V$ to $M$ has $\overline{V_{M}}=\overline{V_{L}}$ and $\Gamma_{V_{M}}=$ $\Gamma_{V_{K}}$. By [R, 29.9, 29.16], $S \otimes_{F} T=(M, G \times H, h)$ where $h$ is the 2-cocycle defined by $h\left(\left(\sigma_{1}, \tau_{1}\right),\left(\sigma_{2}, \tau_{2}\right)\right)=f\left(\sigma_{1}, \sigma_{2}\right) g\left(\tau_{1}, \tau_{2}\right)$. Let $(M, G \times H, h)=$ $\bigoplus_{(\sigma, \tau) \in G \times H} M z_{\sigma \tau}$ and define $w^{\prime}$ on $S \otimes_{F} T=\bigoplus_{(\sigma, \tau) \in G \times H} M z_{\sigma \tau}$ as in Theorem 2.1. Then $w^{\prime}\left(z_{\sigma \tau}\right)=w\left(x_{\sigma}\right)+w\left(y_{\tau}\right)$. To see this,

$$
\begin{aligned}
w^{\prime}\left(z_{\sigma \tau}\right) & =\frac{1}{n m} \sum_{i=1}^{n m} w\left(h\left((\sigma, \tau)^{i}\right), h(\sigma, \tau)\right)=\frac{1}{n m} \sum_{i=1}^{n m} w\left(f\left(\sigma^{i}, \sigma\right) g\left(\tau^{i}, \tau\right)\right) \\
& =\frac{1}{n m} \sum_{i=1}^{n m} w\left(f\left(\sigma^{i}, \sigma\right)\right)+\frac{1}{n m} \sum_{i=1}^{n m} w\left(g\left(\tau^{i}, \tau\right)\right) \\
& =\frac{1}{n} \sum_{i=1}^{n} w\left(f\left(\sigma^{i}, \sigma\right)\right)+\frac{1}{m} \sum_{i=1}^{m} w\left(g\left(\tau^{i}, \tau\right)\right)=w\left(x_{\sigma}\right)+w\left(y_{\tau}\right)
\end{aligned}
$$


since $|G|=n$ and $|H|=m$. The kernel of the canonical map $\mathscr{G}(M / F) \rightarrow$ $\mathscr{G}\left(\overline{V_{M}} / \bar{V}\right)$ is $H$. Let $I$ be the kernel of $\alpha: G \times H \rightarrow \Delta / \Gamma_{V_{M}}=\Delta / \Gamma_{V_{K}}$ given by $\alpha(\sigma, \tau)=w^{\prime}\left(z_{\sigma \tau}\right)+\Gamma_{V_{M}}$. If $\tau \in H \cap I, w^{\prime}\left(z_{1 \tau}\right)+\Gamma_{V_{M}}=w\left(y_{\tau}\right)+\Gamma_{V_{M}}=0$, so $w\left(y_{\tau}\right) \in \Gamma_{V_{M}}=\Gamma_{V_{K}}$, which implies $\tau=1$. Thus $I \cap H=0$, so by Corollary 2.4, Theorem 2.1 applies.

Let $B \subseteq S \otimes_{F} T$ be the integral Dubrovin valuation ring obtained from $w^{\prime}$. Then $\Gamma_{B} / \Gamma_{V_{K}}=\operatorname{im}(\alpha)=\left(\Gamma_{B_{S}}+\Gamma_{B_{T}}\right) / \Gamma_{V_{K}}$, so $\Gamma_{B}=\Gamma_{B_{S}}+\Gamma_{B_{T}} . B / V$ is defectless since $V_{M} / V$ is defectless. Let $\bar{I}$ be the image of $I$ in $\mathscr{G}\left(\overline{V_{M}} / \bar{V}\right)=G$. Then $\bar{B}=\left(\overline{V_{M}}, \bar{I}, k\right)$ for some cocycle $k$. Since $\bar{I}=\left\{\sigma \in G \mid w\left(x_{\sigma}\right) \in\right.$ $\left.\Gamma_{B_{T}}\right\} \supseteq\left\{\sigma \in G \mid w\left(x_{\sigma}\right) \in \Gamma_{V_{L}}\right\}=\mathscr{G}\left(\overline{V_{L}} / Z\left(\overline{B_{S}}\right)\right), Z(\bar{B})=\mathscr{F}(\bar{I}) \subseteq Z\left(\overline{B_{S}}\right)$. $\theta_{B_{S}}\left(w\left(x_{\sigma}\right)\right)=\left.\bar{\sigma}\right|_{Z\left(\overline{B_{S}}\right)}$ and $\theta_{B}\left(w^{\prime}\left(z_{\sigma \tau}\right)\right)=\left.\overline{(\sigma, \tau)}\right|_{Z(\bar{B})}=\left.\bar{\sigma}\right|_{Z(\bar{B})}$. Since $w^{\prime}\left(z_{\sigma \tau}\right)=$ $w\left(x_{\sigma}\right)+w\left(y_{\tau}\right), \theta_{B}\left(w\left(x_{\sigma}\right)\right)=\theta_{B}\left(w^{\prime}\left(z_{\sigma 1}\right)\right)=\left.\bar{\sigma}\right|_{Z(\bar{B})}$, which is the restriction of $\theta_{B_{S}}\left(w\left(x_{\sigma}\right)\right)$ to $Z(\bar{B})$. So the diagram commutes. To determine $Z(\bar{B})$ note that $\Gamma_{B_{S}} / \Gamma_{V}=\left\{w\left(x_{\sigma}\right)+\Gamma_{V} \mid \sigma \in G\right\}$ and $\theta_{B_{S}}\left(w\left(x_{\sigma}\right)+\Gamma_{V}\right)=\left.\bar{\sigma}\right|_{Z\left(\overline{B_{S}}\right)}$. So $\theta_{B_{S}}\left(\left(\Gamma_{B_{S}} \cap \Gamma_{B_{T}}\right) / \Gamma_{V}\right)=\left\{\left.\bar{\sigma}\right|_{Z\left(\overline{B_{S}}\right)} \mid w\left(x_{\sigma}\right) \in \Gamma_{B_{T}}\right\}=\left\{\left.\bar{\sigma}\right|_{Z\left(\overline{B_{S}}\right)} \mid \sigma \in \bar{I}\right\}$. Therefore $\mathscr{F}\left(\theta_{B_{S}}\left(\left(\Gamma_{B_{S}} \cap \Gamma_{B_{T}}\right) / \Gamma_{V}\right)\right)=\mathscr{F}(\bar{I}) \cap Z\left(\overline{B_{S}}\right)=\mathscr{F}(\bar{I})=Z(\bar{B})$ since $\mathscr{F}(\bar{I}) \subseteq$ $Z\left(\overline{B_{S}}\right)$. Finally to see $\Gamma_{B_{T}}=\operatorname{ker}\left(\theta_{B}\right)$, since $\Gamma_{B_{T}}=\Gamma_{V_{K}}+\left\langle w\left(y_{\tau}\right) \mid \tau \in H\right\rangle$ and $\theta_{B}\left(w\left(y_{\tau}\right)+\Gamma_{V}\right)=\mathrm{id}, \Gamma_{B_{T}} \subseteq \operatorname{ker}\left(\theta_{B}\right)$. For the reverse inclusion, take $\alpha \in \operatorname{ker}\left(\theta_{B}\right)$. Since $\Gamma_{B}=\Gamma_{B_{S}}+\Gamma_{B_{T}}, \alpha=w\left(x_{\sigma}\right)+\gamma$ with $\gamma \in \Gamma_{B_{T}}$. Then $w\left(x_{\sigma}\right) \in \operatorname{ker}\left(\theta_{B}\right)$. So we see from above that $\sigma \in \bar{I}$, hence $w\left(x_{\sigma}\right) \in \Gamma_{B_{T}}$.

Corollary 3.9 [JW, Theorem 6.3]. Let $(F, V)$ be a Henselian valued field, $S$ an inertially split division algebra over $F$ and $T$ a tame, totally ramified division algebra over $F$. If $D \sim S \otimes_{F} T$ and $V_{D}, V_{S}, V_{T}$ are the invariant valuation rings of $D, S, T$ respectively lying over $V$, then $\Gamma_{V_{D}}=\Gamma_{V_{S}}+\Gamma_{V_{T}}$, $\Gamma_{V_{T}} / \Gamma_{V}=\operatorname{ker}\left(\theta_{V_{D}}\right), Z\left(\overline{V_{D}}\right)=\mathscr{F}\left(\theta_{V_{S}}\left(\left(\Gamma_{V_{S}} \cap \Gamma_{V_{T}}\right) / \Gamma_{V}\right)\right)$, and the following diagram is commutative:

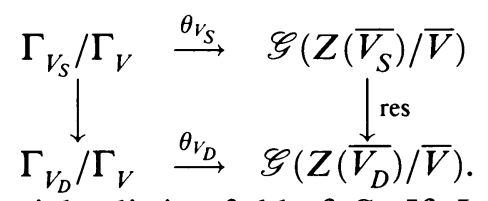

Proof. Say $L_{0}$ is an inertial splitting field of $S$. If $L$ is the normal closure of $L_{0} / F$, then $L / F$ is inertial Galois and $S \sim(L, G, f)$ for some $f \in Z^{2}\left(G, L^{*}\right)$, where $G=\mathscr{G}(L / F)$. Draxl's decomposition theorem [Dr, Theorem 1] shows $T$ is a tensor product of symbol algebras, hence a crossed product. Thus, Theorem 3.8 applies to $(L, G, f) \otimes_{F} T$. Since this algebra is isomorphic to some $M_{k}(D)$, any Dubrovin valuation ring of it lying over $V$ is isomorphic to $M_{k}\left(V_{D}\right)$ by $\left[\mathrm{W}_{1}\right.$, Theorem A]. The corollary then follows because $\overline{M_{k}\left(V_{D}\right)} \cong M_{k}\left(\overline{V_{D}}\right)$ and $\Gamma_{M_{k}\left(V_{D}\right)}=\Gamma_{V_{D}}$ by $\left[\mathrm{W}_{1}\right.$, Corollary 3.5$]$.

In $\left[\mathrm{W}_{2}\right]$ the second author determines those division algebras which arise as the residue division ring of an invariant valuation ring in a finite dimensional $F$ central division algebra. One step in the proof is to decompose a given valued 
division algebra (in the Brauer group $\operatorname{Br}(F)$ ) into a tensor product of other division algebras, such that the residue division ring of each piece can be easily calculated. Given two such pieces, say $D_{1}$ and $D_{2}$, it is seen that $\Gamma_{V_{D_{1}}} \cap \Gamma_{V_{D_{2}}}=$ $\Gamma_{V}$, but $\overline{V_{D_{1}}} \otimes_{\bar{V}} \overline{V_{D_{2}}}$ is not neccesarily a division algebra. However, there is a common subfield $L$ of $D_{1}$ and $D_{2}$ inertial over $F$ with $\overline{V_{L}} \subseteq Z\left(\overline{V_{D_{i}}}\right)$ such that $\overline{V_{D_{1}}} \otimes_{\bar{V}_{L}} \overline{V_{D_{2}}}$ is a division algebra. The second author then proves $\left[\mathrm{W}_{2}\right.$, Proposition 2], a generalization of [ $\mathrm{M}_{1}$, Theorem 1], to describe the underlying division algebra of $D_{1} \otimes_{F} D_{2}$.

Theorem 3.11 below generalizes [ $\mathrm{M}_{2}$, Theorem 3.3] and [ $\mathrm{W}_{2}$, Proposition 2] in the context of Dubrovin valuation rings. The generalization is complicated by the fact that contractions of Dubrovin valuation rings to subalgebras need not be Dubrovin valuation rings. The hypothesis (4) of Theorem 3.11 is needed to go from the original central simple algebra to a centralizer. The examples in $\S 4$ show the pathology that can arise in this situation.

Lemma 3.10. Let $S$ be a central simple F-algebra, $B$ an integral Dubrovin valuation ring over $V$ in $S, L \subset S$ an inertial Galois extension of $F$ with respect to $W / V$. Suppose $\bar{W} \subseteq Z(\bar{B})$ and either $B$ is invariant or for all overrings $V_{i}$ of $V$ in $F, \overline{W V}_{i} \subseteq Z\left(\overline{B V_{i}}\right)$. If $C=C_{S}(L)$ and $A \subseteq C$ is a Dubrovin valuation ring over $W$ then $A$ is integral, $\bar{A}=\bar{B}, \Gamma_{A}=\operatorname{ker}(\rho) \subseteq \Gamma_{B}$, where $\rho: \Gamma_{B} \rightarrow \mathscr{G}(\bar{W} / \bar{V})$ is the map induced by $\theta_{B}$. Furthermore, $A / W$ is defectless iff $B / V$ is defectless.

Proof. If $B$ is invariant then $A=B \cap C$ and the lemma is clear by using [ $\mathrm{M}_{1}$, Theorem 2] and [JW, Lemma 1.8]. So suppose $B$ is not invariant. First, to show $A$ is integral we use [ $\mathrm{W}_{1}$, Theorem F]. Let $B \subseteq B_{i} \subseteq B_{j}$ be two overrings of $B$ with $V_{i}=B_{i} \cap F$ and $V_{j}=B_{j} \cap F$. Since $W / V$ is inertial, $W_{i}=W V_{i}$ and $W_{j}=W V_{j}$ are inertial over $V_{i}$ and $V_{j}$ respectively. Let $A_{i}=A W_{i}$ and $A_{j}=A W_{j}$, overrings of $A$. By Theorem 3.1 we get $Z\left(\overline{A_{j}}\right)=Z\left(\overline{B_{j}}\right) \overline{W_{j}}=$ $Z\left(\overline{B_{j}}\right)$. Let $\widetilde{V}_{i}=V_{i} / J\left(V_{j}\right) \subseteq \bar{V}_{j}$ and $\widetilde{W}_{i}=W_{i} / J\left(W_{j}\right) \subseteq \overline{W_{j}}$. Since $B / V$ is integral, $\widetilde{V}_{i}$ extends uniquely to $Z\left(\overline{B_{j}}\right)$. Thus the diagram below shows $\widetilde{W}_{i}$ extends uniquely to $Z\left(\overline{A_{j}}\right)$. Thus by [W $\mathrm{W}_{1}$, Theorem $\left.\mathrm{F}\right], A / W$ is integral.

$$
\begin{array}{lll} 
& & Z\left(\overline{A_{j}}\right)=Z\left(\overline{B_{j}}\right) \\
\widetilde{W}_{i} & \subset \overline{W_{j}} \\
\cup & \bar{\cup} \\
\widetilde{V}_{i} & \subset & \bar{V}_{j}
\end{array}
$$

To determine $\Gamma_{A}$ and $\bar{A}$ we "Henselize" and use [W ${ }_{1}$, Theorem B]. If $\left(F_{h}, V_{h}\right)$ is the Henselization of $(F, V)$ and $\left(L_{h}, W_{h}\right)$ is the Henselization of $(L, W)$, then $L_{h}=L F_{h}$. Let $D, E$ be the underlying division algebras of $S \otimes_{F} F_{h}$ and $C \otimes_{L} L_{h}$ respectively. Since $F_{h}$ and $L_{h}$ are Henselian, there are invariant valuation rings $R \subseteq D$ and $R^{\prime} \subseteq E$ extending $V_{h}$ and $W_{h}$ respectively. So $\left[\mathrm{W}_{1}\right.$, Theorem B] shows that $\Gamma_{B}=\Gamma_{R}, \Gamma_{A}=\Gamma_{R^{\prime}}, \bar{B} \sim \bar{R}$ 
and $\bar{A} \sim \overline{R^{\prime}}$. By Corollary 3.5, $\overline{R^{\prime}} \sim \bar{D}$, so $\bar{A} \sim \bar{B}$ as $\bar{W} \subseteq Z(\bar{B})$. Also, $\Gamma_{R^{\prime}}=\theta_{R}^{-1}\left(\mathscr{G}\left(Z(\bar{R}) / \overline{W_{h}}\right)\right)$, hence $\Gamma_{A}=\operatorname{ker}(\rho) . \delta(A)=\delta\left(R^{\prime}\right)=\delta(R)=\delta(B)$, so $A$ is defectless iff $B$ is defectless. The only thing remaining is to show $\bar{A}=\bar{B}$. Since $\bar{A} \sim \bar{B}$ it suffices to show $t_{A}=t_{B}$. Since $A$ and $B$ are integral it is then enough to show $n_{A}=n_{B}$. By definition of $n_{A}$ and $n_{B}, S \otimes_{F} F_{h}=$ $M_{n_{B}}(D)$ and $C \otimes_{L} L_{h}=M_{n_{A}}(E)$. Since $C \sim S \otimes_{F} L$, dimension count shows $S \otimes_{F} L=M_{l}(C)$, where $l=[L: F]$. Therefore $\left(S \otimes_{F} L\right) \otimes_{L} L_{h}=M_{l n_{A}}(E)$. But $\left(S \otimes_{F} L\right) \otimes_{L} L_{h}=S \otimes_{F} L_{h}=S \otimes_{F}\left(F_{h} \otimes_{F_{h}} L_{h}\right)=\left(S \otimes_{F} F_{h}\right) \otimes_{F_{h}} L_{h}=M_{n_{B}}\left(D \otimes_{F_{h}} L_{h}\right)$. Since $\overline{W_{h}}=\bar{W} \subseteq Z(\bar{B})=Z(\bar{R})$ and $F_{h}$ is Henselian, $L_{h}$ embeds in $D$, so $D \otimes_{F_{h}} L_{h}=M_{l}(E)$. Thus $M_{l n_{A}}(E)=M_{n_{B} l}(E)$, so $n_{A}=n_{B}$.

Theorem 3.11. Let $S_{1}$ and $S_{2}$ be central simple $F$-algebras, $B_{i} \subseteq S_{i}$ integral Dubrovin valuation rings with $B_{1} \cap F=B_{2} \cap F=V$. Suppose $S_{1}$ and $S_{2}$ contain a common subfield $L$ which is Galois over $F, l=[L: F]$, and $L$ has an extension $W$ of $V$ for which $W / V$ is inertial. Suppose further that

(1) $\bar{W} \subseteq Z\left(\overline{B_{1}}\right), Z\left(\overline{B_{2}}\right)$,

(2) $\Gamma_{B_{1}} \cap \Gamma_{B_{2}}=\Gamma_{V}$,

(3) $\overline{B_{1}} \otimes_{\bar{W}} \overline{B_{2}}$ is simple Artinian,

(4) Either the $B_{i}$ are both invariant, or for all overrings $V_{i} \supseteq V$ in $F$, $\overline{W V_{i}} \subseteq Z\left(\overline{B_{1} V_{i}}\right), Z\left(\overline{B_{2} V_{i}}\right)$,

(5) $B_{1} / V$ is defectless.

If $S=S_{1} \otimes_{F} S_{2}$ and $B$ is a Dubrovin valuation ring in $S$ lying over $V$, then $B$ is integral, $\bar{B}=M_{l}\left(\overline{B_{1}} \otimes_{\bar{W}} \overline{B_{2}}\right), \Gamma_{B}=\left\{\gamma_{1}+\gamma_{2} \in \Gamma_{B_{1}}+\Gamma_{B_{2}} \mid \rho_{1}\left(\gamma_{1}\right)=\rho_{2}\left(\gamma_{2}\right)\right\}$, where $\rho_{i}: \Gamma_{B_{i}} \rightarrow \mathscr{G}(\bar{W} / \bar{V})$ is given by $\rho_{i}\left(b B_{i}^{*}\right)(\bar{w})=\overline{b w b^{-1}}$.

Proof. Let $C_{i}=C_{S_{i}}(L)$ and $A_{i}$ Dubrovin over $W$ in $C_{i}$. By Lemma 3.10, $A_{i}$ is integral over $W, \overline{A_{i}}=\overline{B_{i}}$ and $\Gamma_{A_{i}}=\Gamma_{B_{i}}$. Thus $\Gamma_{A_{1}} \cap \Gamma_{A_{2}}=\Gamma_{V}=\Gamma_{W}$ and $\overline{A_{1}} \otimes_{\bar{W}} \overline{A_{2}}$ is simple. Furthermore $A_{1} / W$ is defectless since $B_{1} / V$ is defectless. By $\left[\mathrm{M}_{2}\right.$, Theorem 3.3] if $C=C_{1} \otimes_{L} C_{2}$ and $A$ is the integral Dubrovin valuation ring of $C$ described by that theorem, $\Gamma_{A}=\Gamma_{A_{1}}+\Gamma_{A_{2}}$ and $\bar{A}=\overline{A_{1}} \otimes_{\bar{W}} \overline{A_{2}}$. We write $S_{i}=\left(C_{i}, G,\left(\omega_{i}, f_{i}\right)\right)$, with $\left(\omega_{i}, f_{i}\right)$ chosen as in Theorem 2.1 so that $f_{i}(\sigma, \tau) \in \operatorname{st}\left(A_{i}\right)$ for all $\sigma, \tau \in G$. By the product theorem [T, Theorem 1.6] or [J $\left.{ }_{2}, 1.15\right]$ or [KY, Theorem 3], $S=S_{1} \otimes_{F} S_{2} \sim$ $\left(C, G,\left(\omega_{1} \otimes \omega_{2}, f_{1} \otimes f_{2}\right)\right)$. Let $S_{0}=\left(C, G,\left(\omega_{1} \otimes \omega_{2}, f_{1} \otimes f_{2}\right)\right)$. By dimension count, $S=M_{l}\left(S_{0}\right)$ and $S_{0} \otimes_{F} L=M_{l}(C)$, so $S \otimes_{F} L=M_{l^{2}}(C)$. Since $S \otimes_{F} L$ contains the integral Dubrovin valuation ring $M_{l^{2}}(A), S$ contains an integral Dubrovin valuation ring $B$ by Theorem 3.1. As $S=M_{l}\left(S_{0}\right), S_{0}$ contains an integral Dubrovin valuation ring $B_{0}$ with $B \cong M_{l}\left(B_{0}\right)$. That corollary gives $\bar{A} \sim M_{l^{2}}(\bar{A}) \sim \bar{B} \otimes_{Z(\bar{B})} Z(\bar{B}) \bar{W}=\bar{B}$ as $\bar{W} \subseteq Z(\bar{B})$. Because

$$
\Gamma_{A}=\theta_{B}^{-1}(\mathscr{G}(Z(\bar{B}) /(Z(\bar{B}) \cap \bar{W})))=\theta_{B}^{-1}(\mathscr{G}(Z(\bar{B}) / \bar{W})),
$$

$\left|\Gamma_{B}: \Gamma_{A}\right|=l$. As $\delta(A)=\delta(B)$, using [ $\mathrm{W}_{1}$, Theorem $\left.\mathrm{C}\right]$ applied to $[S: F]$ and $[C: L]$ we see that $[\bar{B}: \bar{V}]=l^{2}[\bar{A}: \bar{V}]$, so $\bar{B}=M_{l}(\bar{A})=M_{l}\left(\overline{B_{1}} \otimes_{\bar{W}} \overline{B_{2}}\right)$. 
To determine $\Gamma_{B}$, we have to look more closely at $S_{0}$. Say $S_{1}=\bigoplus_{\sigma \in G} C x_{\sigma}$, $S_{2}=\bigoplus_{\sigma \in G} C y_{\sigma}$ and $S_{0}=\bigoplus_{\sigma \in G} C z_{\sigma}$ where the $x_{\sigma}, y_{\sigma}, z_{\sigma}$ correspond to $\left(\omega_{1}, f_{1}\right),\left(\omega_{2}, f_{2}\right)$ and $\left(\omega_{1} \otimes \omega_{2}, f_{1} \otimes f_{2}\right)$ respectively. Since $f_{i}(\sigma, \tau) \in \operatorname{st}\left(A_{i}\right)$, $f_{1}(\sigma, \tau) \otimes f_{2}(\sigma, \tau) \in \operatorname{st}(A)$. If $w_{1}, w_{2}, w$ are the value functions on $C_{1}, C_{2}$, $C$ corresponding to $A_{1}, A_{2}, A$ and $w_{1}^{\prime}, w_{2}^{\prime}, w^{\prime}$ are the value functions on $S_{1}, S_{2}, S_{0}$ corresponding to $B_{1}, B_{2}, B_{0}$ respectively, then

$$
\begin{aligned}
w^{\prime}\left(z_{\sigma}\right) & =\frac{1}{l} \sum_{i=1}^{l} w\left(h\left(\sigma^{i}, \sigma\right)\right)=\frac{1}{l} \sum_{i=1}^{l} w\left(f_{1}\left(\sigma^{i}, \sigma\right) \otimes f_{2}\left(\sigma^{i}, \sigma\right)\right) \\
& =\frac{1}{l} \sum_{i=1}^{l} w_{1}\left(f_{1}\left(\sigma^{i}, \sigma\right)\right)+\frac{1}{l} \sum_{i=1}^{l} w_{2}\left(f_{2}\left(\sigma^{i}, \sigma\right)\right) \\
& =w_{1}^{\prime}\left(x_{\sigma}\right)+w_{2}^{\prime}\left(y_{\sigma}\right) .
\end{aligned}
$$

Therefore $\Gamma_{B}=\Gamma_{A}+\left\langle w^{\prime}\left(z_{\sigma}\right)\right\rangle \subseteq\left(\Gamma_{A_{1}}+\left\langle w_{1}\left(x_{\sigma}\right)\right\rangle\right)+\left(\Gamma_{A_{2}}+\left\langle w_{2}\left(y_{\sigma}\right)\right\rangle\right)=\Gamma_{B_{1}}+\Gamma_{B_{2}}$. Since $\rho_{1}\left(w_{1}\left(x_{\sigma}\right)\right)=\rho_{2}\left(w_{2}\left(y_{\sigma}\right)\right)=\bar{\sigma}$ it follows that $\Gamma_{B}=\left\{\gamma_{1}+\gamma_{2} \in \Gamma_{B_{1}}+\Gamma_{B_{2}} \mid\right.$ $\left.\rho_{1}\left(\gamma_{1}\right)=\rho_{2}\left(\gamma_{2}\right)\right\}$.

This result has a couple of corollaries which deal with invariant valuation rings and arbitrary Dubrovin valuation rings. Note that $\overline{B_{1}} \otimes_{\bar{W}} \overline{B_{2}}$ being simple is equivalent to $Z\left(\overline{B_{1}}\right)$ and $Z\left(\overline{B_{2}}\right)$ linearly disjoint over $\bar{W}$.

Corollary 3.12. Suppose $(F, V)$ is Henselian, $D_{1}, D_{2} F$-central division algebras, $V_{D_{i}} \subseteq D_{i}$ invariant valuation rings lying over $V$ with $V_{D_{1}} / V$ defectless, $\Gamma_{V_{D_{1}}} \cap \Gamma_{V_{D_{2}}}=\Gamma_{V}, \mathscr{L}=Z\left(\overline{V_{D_{1}}}\right) \cap Z\left(\overline{V_{D_{2}}}\right)$ separable over $\bar{V}$ and $Z\left(\overline{V_{D_{1}}}\right)$ and $Z\left(\overline{V_{D_{2}}}\right)$ linearly disjoint over $\mathscr{L}$. If $D$ is the underlying division algebra of $D_{1} \otimes_{F} D_{2}$, then $\Gamma_{V_{D}}=\left\{\gamma_{1}+\gamma_{2} \in \Gamma_{V_{D_{1}}}+\Gamma_{V_{D_{2}}} \mid \rho_{1}\left(\gamma_{1}\right)=\rho_{2}\left(\gamma_{2}\right)\right\}$ where $\rho_{i}: \Gamma_{V_{D_{i}}} \rightarrow \mathscr{G}(\mathscr{L} / \bar{V})$ is as above and $\overline{V_{D}} \sim \overline{V_{D_{1}}} \otimes_{\mathscr{L}} \overline{V_{D_{2}}}$.

Proof. Because $\mathscr{L}$ is separable over $\bar{V}$ and $F$ is Henselian, there is a unique (up to isomorphism) inertial extension $L$ of $F$ with $\overline{V_{L}}=\mathscr{L}$, which lies in both $D_{1}$ and $D_{2}$. As $Z\left(\overline{V_{D_{1}}}\right)_{\text {sep }}$ is abelian Galois over $\bar{V}$ by [JW, Proposition 1.7] and $\mathscr{L} \subseteq Z\left(\overline{V_{D_{1}}}\right)_{\text {sep }}, \mathscr{L} / \bar{V}$ is Galois, hence $L / F$ is Galois. The linear disjointness of $Z\left(\overline{V_{D_{1}}}\right)$ and $Z\left(\overline{V_{D_{2}}}\right)$ over $\mathscr{L}$ implies that $\overline{V_{D_{1}}} \otimes \overline{V_{D_{2}}}$ is simple. Since $D_{1} \otimes_{F} D_{2} \cong M_{n}(D)$ for some $D$, the corollary then follows directly from Theorem 3.11 and $\left[\mathrm{W}_{1}\right.$, Corollary 3.6].

Corollary 3.13. Let $(F, V)$ be any valued field, $S_{1}$ and $S_{2}$ central simple $F$ algebras and $B_{i} \subseteq S_{i}$ Dubrovin valuation rings over $V$. Suppose $B_{1} / V$ is defectless, $\Gamma_{B_{1}} \cap \Gamma_{B_{2}}=\Gamma_{V}, \mathscr{L}=Z\left(\overline{B_{1}}\right) \cap Z\left(\overline{B_{2}}\right)$ separable over $\bar{V}$, and $Z\left(\overline{B_{1}}\right)$ and $Z\left(\overline{B_{2}}\right)$ linearly disjoint over $\mathscr{L}$. Then if $B$ is a Dubrovin valuation ring in $S_{1} \otimes_{F} S_{2}$ lying over $V$, we have $\Gamma_{B}=\left\{\gamma_{1}+\gamma_{2} \mid \rho_{1}\left(\gamma_{1}\right)=\rho_{2}\left(\gamma_{2}\right)\right\}$ and $\bar{B} \sim \overline{B_{1}} \otimes_{\mathscr{L}} \overline{B_{2}}$.

Proof. This follows easily from Corollary 3.12 and the Henselization theorem $\left[\mathrm{W}_{1}\right.$, Theorem B]. 
The conditions in Corollaries 3.12 and 3.13 that $Z\left(\overline{B_{1}}\right) \cap Z\left(\overline{B_{2}}\right)$ is separable over $V$ and $Z\left(\overline{B_{1}}\right)$ and $Z\left(\overline{B_{2}}\right)$ be linearly disjoint over the $Z\left(\overline{B_{1}}\right) \cap Z\left(\overline{B_{2}}\right)$ occur quite often. For instance if either $Z\left(\overline{B_{1}}\right)$ or $Z\left(\overline{B_{2}}\right)$ is separable over $\bar{V}$ then both conditions hold. Hence if $B_{1} / V$ is tame then these conditions hold.

\section{EXAMPLES}

In Theorem 3.11 it is somewhat unsatisfying to have to include hypothesis (4). However, the second example below will show that this is necessary. The difficulty shows up in Lemma 3.10. Given $C$ a subalgebra of a central simple algebra $S$ and an integral Dubrovin valuation ring $B$ in $S$ one would like to obtain an integral Dubrovin valuation ring in $C$. This is not always possible, as shown in the next example. This shows one particular difficulty with Dubrovin valuation rings, that contractions of Dubrovin valuation rings to subalgebras need not be Dubrovin.

Example 4.1. $D$ an F-central division algebra, $L \subset D$ a Galois inertial field extension of $F$ with respect to the valuation rings $W / V, B$ integral Dubrovin in $D$ lying over $V$ with $\bar{W} \subseteq Z(\bar{B}), C=C_{D}(L)$, but $C$ contains no integral Dubrovin valuation ring lying over $W$. Thus $B \cap C$ is not a Dubrovin valuation ring.

Proof. Let

$$
F=\mathbf{Q}(x, y), \quad L=F(\sqrt{2}), \quad D=\left(\frac{2,1+x}{F}\right) \otimes_{F}\left(\frac{3, y}{F}\right) .
$$

Let $V$ be composite of the usual rank two valuation ring on $F$ with the 5adic valuation ring on $\mathbf{Q}$. (That is, $V$ is the valuation ring inherited from $\mathbf{Q}((x))((y))$.) Since 2 is not a square $\bmod 5, \mathbf{Z}_{(5)}$ extends uniquely to $\mathbf{Q}(\sqrt{2})$, hence $V$ extends uniquely to an inertial valuation ring $W$ of $L . D$ can be seen to be a division ring in much the same way as in the examples in $\left[\mathrm{M}_{2}\right]$ (by considering the $y$-adic valuation ring of $F$ ), but since knowing whether $D$ is a division ring is not needed, the proof will be omitted.

Now $\left(\frac{2,1+x}{V}\right)$ is an Azumaya algebra over $V$ with residue ring $\left(\frac{2,1}{\bar{V}}\right) \cong$ $M_{2}(\mathbf{Z} / 5 \mathbf{Z})$. By [JW, Ex. 4.3] $\left(\frac{3, y}{F}\right)$ is a nicely semiramified division algebra over $F$ with residue division algebra $\bar{F}(\sqrt{3})=(\mathbf{Z} / 5 \mathbf{Z})(\sqrt{3})$. If $B$ is the tensor product of the above Azumaya algebra with this invariant valuation ring, then $B$ is an integral Dubrovin valuation ring over $V$ with residue ring $M_{2}(\mathbf{Z} / 5 \mathbf{Z}) \otimes_{\mathbf{Z} / 5 \mathbf{Z}}$ $(\mathbf{Z} / 5 \mathbf{Z})(\sqrt{3})=M_{2}((\mathbf{Z} / 5 \mathbf{Z})(\sqrt{3}))=M_{2}((\mathbf{Z} / 5 \mathbf{Z})(\sqrt{2}))$ by $\left[\mathbf{M}_{2}\right.$, Theorems 3.3 and 3.4]. Thus $\bar{W}=(\mathbf{Z} / 5 \mathbf{Z})(\sqrt{2}) \subseteq Z(\bar{B})$.

Let $C=C_{D}(L)=L \otimes_{F}\left(\frac{3, y}{F}\right)=\left(\frac{3, y}{L}\right)$. If $W^{\prime}$ is the usual rank two valuation ring on $L$ with residue field $\mathbf{Q}(\sqrt{2})$, then $C$ is a nicely semiramified division algebra over $L$ with residue division algebra $\overline{W^{\prime}}(\sqrt{3})=\mathbf{Q}(\sqrt{2}, \sqrt{3})$. Now the 5 -adic valuation ring of $\mathbf{Q}$ extends in two ways to $\mathbf{Q}(\sqrt{2}, \sqrt{3})$. Let $A$ be the composite of the nicely semiramified valuation ring in $C$ with one of the 
extensions of $\mathbf{Z}_{(5)}$ to $\mathbf{Q}(\sqrt{2}, \sqrt{3})$. By [W $\mathbf{W}_{1}$, Th. F], since $\mathbf{Z}_{(5)}$ extends in more than one way to $\mathbf{Q}(\sqrt{2}, \sqrt{3}), A$ is not integral. This shows that the inequality $s_{B} \leq s_{A}$ in Theorem 3.1 can be strict.

As a preliminary to the next example, for $F, L, V, W$ as in the above example, let $D=\left(\frac{2, x}{F}\right) \otimes_{F}\left(\frac{3, y}{F}\right)$ and $V^{\prime}$ the usual rank two valuation ring of $F$ with residue field $\mathbf{Q}$. By [JW, Ex. 4.3], $\left(\frac{2, x}{F}\right)$ and $\left(\frac{3, y}{F}\right)$ are nicely semiramified division algebras over $V^{\prime}$ with value groups $\frac{1}{2} \mathbf{Z} \times \mathbf{Z}, \mathbf{Z} \times \frac{1}{2} \mathbf{Z}$ and residue division algebras $\mathbf{Q}(\sqrt{2}), \mathbf{Q}(\sqrt{3})$ respectively. Thus by [JW, Ex. 4.3], $D$ is a nicely semiramified division algebra whose invariant valuation ring $B^{\prime}$ satisfies $\overline{B^{\prime}}=\mathbf{Q}(\sqrt{2}, \sqrt{3}) . \mathbf{Z}_{(5)}$ extends in two ways to $\overline{B^{\prime}}$. If $B$ is the composite of $B^{\prime}$ and one of these extensions, then $B$ is a noninvariant Dubrovin valuation ring of $D$ over $V$ with $t_{B}=1$. Thus $n_{B} \neq t_{B}$, so $B$ is not integral. We will use this to give the following example.

Example 4.2. $D_{1}, D_{2}$ F-central division algebras, $B_{i} \subseteq D_{i}$ integral Dubrovin over $V, L \subset D_{1}, D_{2}$ inertial Galois over $F$ (with respect to $W \subseteq L$ ) with $\Gamma_{B_{1}} \cap \Gamma_{B_{2}}=\Gamma_{V}, \bar{W} \subseteq Z\left(\overline{B_{i}}\right), \overline{B_{1}} \otimes_{\bar{W}} \overline{B_{2}}$ simple and $B_{1} / V$ defectless, but there is no integral Dubrovin valuation ring in $D_{1} \otimes_{F} D_{2}$ lying over $V$.

Proof. Let $F, L, V, W$ be as above, and set

$$
D_{1}=\left(\frac{2,11}{F}\right) \otimes_{F}\left(\frac{3,7 y}{F}\right), \quad D_{2}=\left(\frac{2,11 x}{F}\right) \otimes_{F}\left(\frac{3,7}{F}\right) .
$$

Thus $L \subseteq D_{i} \cdot\left(\frac{2,11}{F}\right)$ and $\left(\frac{3,7}{F}\right)$ contain Azumaya algebras $A_{1}$ and $A_{2}$ respectively with $\overline{A_{1}}=\overline{A_{2}}=M_{2}(\mathbf{Z} / 5 \mathbf{Z})$. The algebras $\left(\frac{3,7 y}{F}\right)$ and $\left(\frac{2,11 x}{F}\right)$ contain nicely semiramified invariant valuation rings $C_{1}$ and $C_{2}$ respectively with $\Gamma_{C_{1}}=\mathbf{Z} \times \frac{1}{2} \mathbf{Z} \times \mathbf{Z}, \Gamma_{C_{2}}=\mathbf{Z} \times \mathbf{Z} \times \frac{1}{2} \mathbf{Z}$ and $\overline{C_{1}}=\overline{C_{2}}=\bar{W}$ respectively, as $(\mathbf{Z} / 5 \mathbf{Z}(\sqrt{2}))=(\mathbf{Z} / 5 \mathbf{Z}(\sqrt{3}))=\bar{W}$. If $B_{i}=A_{i} \otimes_{V} C_{i}$, then by $\left[\mathrm{M}_{2}\right.$, Theorems 3.3 and 3.4], $B_{i}$ is integral Dubrovin, $\overline{B_{i}}=M_{2}(\bar{W})$ and $\Gamma_{B_{i}}=\Gamma_{C_{i}}$. So all the above conditions hold. However

$$
\begin{aligned}
D_{1} \otimes_{F} D_{2} & \cong\left(\frac{2,11}{F}\right) \otimes_{F}\left(\frac{2,11 x}{F}\right) \otimes_{F}\left(\frac{3,7 y}{F}\right) \otimes_{F}\left(\frac{3,7}{F}\right) \\
& \cong M_{2}\left(\left(\frac{2, x}{F}\right)\right) \otimes_{F} M_{2}\left(\left(\frac{3, y}{F}\right)\right) \cong M_{4}(D)
\end{aligned}
$$

where $D$ is the division algebra described before this example. Since $D$ has no integral Dubrovin valuation ring over $V$, neither does $D_{1} \otimes_{F} D_{2}$.

\section{REFERENCES}

[BG] H. H. Brungs and J. Gräter, Extensions of valuation rings in central simple algebras, Trans. Amer. Math. Soc. (to appear).

[Dr] P. Draxl, Ostrowski's theorem for Henselian valued skew fields, J. Reine Angew. Math. 354 (1984), 213-218. 
[D 1 ] N. I. Dubrovin, Noncommutative valuation rings, Trudy Moskov. Mat. Obshch. 45 (1982), 265-280; English transl., Trans. Moscow Math. Soc. 45 (1984), 273-287.

$\left[\mathrm{D}_{2}\right]$ N. I. Dubrovin, Noncommutative valuation rings in simple finite-dimensional algebras over a field, Mat. Sb. 123 (165) (1984), 496-509; English transl., Math. USSR-Sb. 51 (1985), 493505.

[JW] B. Jacob and A. Wadsworth, Division algebras over Henselian fields, J. Algebra (to appear).

[ $\left.\mathrm{J}_{1}\right]$ W. Jehne, Idelklassenfaktorensysteme und verallgemeinerte Theorie der verschränkten Produkte, Abh. Math. Sem. Hamburg 18 (1952), 70-98.

$\left[\mathrm{J}_{2}\right]$ W. Jehne, Separable adel algebras and a presentation of Weil groups, J. Reine Angew. Math. 375/376 (1987), 211-237.

[KY] V. V. Kursov and V. I. Yanchevskii, Crossed products over simple algebras and their automorphism groups, Akad. Nauk Belo. Inst. Math., preprint \# 35, Minsk, 1987 (Russian).

[M $\mathrm{M}_{1}$ ] P. Morandi, The Henselization of a valued division algebra, J. Algebra 122 (1989), 232-243.

$\left[\mathrm{M}_{2}\right] \ldots$, Value functions on central simple algebras, Trans. Amer. Math. Soc. 315 (1989), 605622.

[R] I. Reiner, Maximal orders, Academic Press, London, 1975.

[T] J.-P. Tignol, Generalized crossed products, Séminaire Mathématique (nouvelle série), Rapport No. 106, Université Catholique de Louvain, Louvain-la-Neuve, Belgium, 1987.

[W ${ }_{1}$ ] A. Wadsworth, Dubrovin valuation rings and Henselization, Math. Ann. 283 (1989), 301-328.

$\left[\mathrm{W}_{2}\right]$ _ The residue division ring of a valued division algebra, Bull. Soc. Math. Belg. Ser. A 40 (1988), 307-322.

Department of Mathematics, University of California, San Diego, la Jolla, CaliforNIA 92093 (Current address of A. R. Wadsworth)

Current address (P. J. Morandi): Department of Mathematics, University of Texas, Austin, Texas 78712 